\title{
Maintenance of Response After Open-Label Treatment with Atomoxetine Hydrochloride in International European and Non-European Adult Outpatients with Attention-Deficit/Hyperactivity Disorder: A Placebo-Controlled, Randomised Withdrawal Study
}

Himanshu Upadhyaya, MBBS, MS*

J. Antoni Ramos-Quiroga, MD, PhD** Lenard A. Adler, MD***

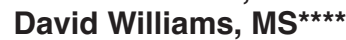
Yoko Tanaka, PhD* Jeannine R. Lane, $\mathrm{PhD}^{\star \star \star \star}$ Rodrigo Escobar, MD*

Paula Trzepacz, MD* Angelo Camporeale, MD* Albert J. Allen, MD, PhD*

* Lilly Research Laboratories, Lilly Corporate Center, Indianapolis, USA

** Department of Psychiatry, Hospital Universitari Vall d'Hebron, CIBERSAM, Universitat Autònoma de Barcelona, Barcelona, Spain

*** New York University School of Medicine, New York University Medical Center and NY VA Harbor Healthcare Service, New York, USA **** inVentiv Health Clinical, Indianapolis, USA

USA

SPAIN 
Methods: Patients ( $\mathrm{N}=2017), 18$ to 50 years of age, diagnosed with ADHD from 152 outpatient sites in 18 countries received 12 weeks of open-label atomoxetine (40-100 $\mathrm{mg} /$ day) followed by 12 weeks of double-blind maintenance ( 80 or $100 \mathrm{mg} /$ day). Responders were randomized to atomoxetine $(\mathrm{N}=266)$ or placebo $(\mathrm{N}=258)$ for 25 -weeks of double-blind treatment. The percentage of patients with a reduction $\geq 30 \%$ in their baseline Conners' ADHD Rating Scale Investigator-Rated: Screening Version (CAARS-Inv:SV) total score and a score of $\leq 3$ on the Clinical Global Impression ADHD-Severity (CGIADHD-S) after 25 weeks was compared between treatment groups with a Fisher's exact test. Mean changes from baseline in the CAARS-Inv:SV and Adult Attention-Deficit/Hyperactivity Disorder Quality of Life (AAQoL) were analysed.

Results: Most patients enrolled (60\%) were from Europe. More atomoxetine- than placebo-treated patients maintained a satisfactory response postrandomisation $(64.3 \%$ vs. $50.0 \%$; $p<.001)$. Time-to-relapse was significantly longer for atomoxetine than placebo $(p=.004)$. Atomoxetine maintained greater improvements in ADHD symptoms compared with placebo (LS mean worsening in the CAARS-Inv:SV total score was 0.9 vs. $4.8[p<.001]$ and in the CGI-ADHD-S rating was 0.0 vs. $0.5[p<.001])$. These results were supported by self- or observer-rated measures. Lastly, atomoxetine maintained greater improvements in quality of life compared with placebo (AAQoL total score was 0.4 vs. $-4.0 ; p=.002$ ).

Conclusions: This study demonstrated that atomoxetine was superior to placebo in maintaining significantly greater treatment responses for up to 1 year in adults with ADHD.

\section{Introduction}

The estimated national average prevalence of attention-deficit hyperactivity disorder (ADHD) in adulthood is $3.4 \%$ (range $1.2 \%$ $7.3 \%$ ), with the prevalence in Europe ranging from $1.2 \%$ to $7.3 \%$ and in non-European countries ranging from $1.9 \%$ to $5.2 \%{ }^{1}$. The burden of ADHD in adult patients is substantial and is often characterised by academic or occupational impairment and dysfunction within the family and the society ${ }^{2-4}$. Clinical manifestations include inability to concentrate; lack of organisation; inability to establish and maintain a routine; poor discipline; low self-esteem; forgetfulness or poor memory; and confusion or trouble thinking clearly $^{2-4}$. Poor impulse control may also be present, and can result in traffic accidents and high alcohol and drug abuse comorbidity ${ }^{5,6}$.
A variety of pharmacotherapies are available to treat ADHD in pediatric patients, and those that are effective in children generally seem effective in adults ${ }^{7-14}$. Although not approved in most European countries, the most widely used pharmacological treatments for ADHD in adults are the stimulants methylphenidate (MPH) and dexamphetamine ${ }^{15}$. Considerable interest in alternative, nonstimulant therapies exists, because some patients respond poorly to stimulants or are unable to tolerate them, and physicians with concerns about misuse in a population at increased risk for substance abuse are reluctant to use them ${ }^{16,17}$.

Atomoxetine hydrochloride, a selective norepinephrine reuptake inhibitor, is a nonstimulant medication approved for the treatment of ADHD in pediatric patients in multiple countries and in adults in a growing number of countries (e.g., the United States, 
Mexico, Canada, Japan, and Australia) ${ }^{18}$. The beneficial effects of atomoxetine on the core symptoms of ADHD have been demonstrated in numerous studies in children ${ }^{19-25}$, and in an increasing number of studies in adults ${ }^{7,9,10}$, even those with comorbid alcohol use and social anxiety disorders ${ }^{26,27}$. Although there have been studies examining the efficacy and safety of atomoxetine in adults with ADHD for up to 6 months $^{7}$, none have evaluated maintenance of response in adults using a randomized withdrawal design. In the current study, maintenance of response to atomoxetine was evaluated using a 3-period randomised withdrawal design in adults with ADHD who responded to 12 weeks of atomoxetine during an open-label treatment period and maintained that response during a 12-week double-blind maintenance period. Maintenance of response was evaluated in atomoxetine responders during a final 25week, double-blind, placebo-controlled, randomised withdrawal period.

\section{Method}

\section{Participants}

This study was conducted at 152 centers in 18 countries. Per the protocol, $60 \%$ of patients were enrolled from countries in Europe. Patients were enrolled from Argentina $(\mathrm{N}=35)$, Austria ( $\mathrm{N}=114)$, Belgium ( $\mathrm{N}=151)$, Canada $(\mathrm{N}=82)$, Denmark $(\mathrm{N}=19)$, Finland $(\mathrm{N}=76)$, France $(\mathrm{N}=73)$, Germany $(\mathrm{N}=495)$, Italy $(\mathrm{N}=39)$, Mexico $(\mathrm{N}=61)$, Netherlands $(\mathrm{N}=82)$, Portugal $(\mathrm{N}=6)$, Russia $(\mathrm{N}=7)$, Spain $(N=195)$, Sweden $(N=147)$, Switzerland $(\mathrm{N}=10)$, United Kingdom $(\mathrm{N}=34)$, and the United States $(\mathrm{N}=908)$.

Eligible patients were aged 18 to 50 years, met the Diagnostic and Statistical Manual of Mental Disorders, Fourth Edition, Text Revi- sion $^{\mathrm{TM}}$ (DSM-IV-TR) criteria for current and childhood ADHD as assessed by the Conners' Adult ADHD Diagnostic Interview for DSM-IV ${ }^{28}$; had a score of $\geq 2$ on at least six items of either the inattentive or hyperactive core subscales of the Conners' Adult Attention-Deficit/ Hyperactivity Disorder Rating Scale (CAARS)-Investigator Screening Version (CAARS-Inv:SV) ${ }^{28}$ with adult ADHD prompts for current symptoms and of the CAARS-Observer Screening Version (CAARS$\mathrm{O}: \mathrm{SV})^{28}$; and, had a score of $\geq 20$ on CAARSInv:SV 18-item total ADHD symptom score. Patients were also required to have a Clinical Global Impression-ADHD-Severity (CGIADHD-S) ${ }^{29}$ rating of $\geq 4$ (moderately ill) at Visits 1 and 2. The CAARS and CGI-ADHD$\mathrm{S}$ scales are described in greater detail below.

Patients who met DSM-IV-TR diagnostic criteria for any history of bipolar disorder, current major depression, a current anxiety disorder (including generalised anxiety disorder, panic disorder, or social phobia), or any history of a psychotic disorder were excluded from the study. Patients who were currently using alcohol, drugs of abuse, or any prescribed or over-the-counter medication in a manner that the investigator considered indicative of chronic abuse or who met DSM-IV-TR criteria for alcohol or other substance dependence were also excluded.

An informed consent document approved by an ethical review board or similar body was signed by the patient, and deemed appropriate, according to local laws and regulations. The study was conducted in accordance with the Declaration of Helsinki and the applicable laws and regulations of the study countries and regions.

\section{Study Design}

In this study, a randomised, double-blind, maintenance of response (randomised withdrawal) design was implemented to test the hy- 
pothesis that atomoxetine ( 80 or $100 \mathrm{mg} /$ day) was superior to placebo in maintaining a satisfactory response at the end of double-blind treatment in adult outpatients with ADHD. The study design was made up of three study periods and is illustrated in Figure 1.

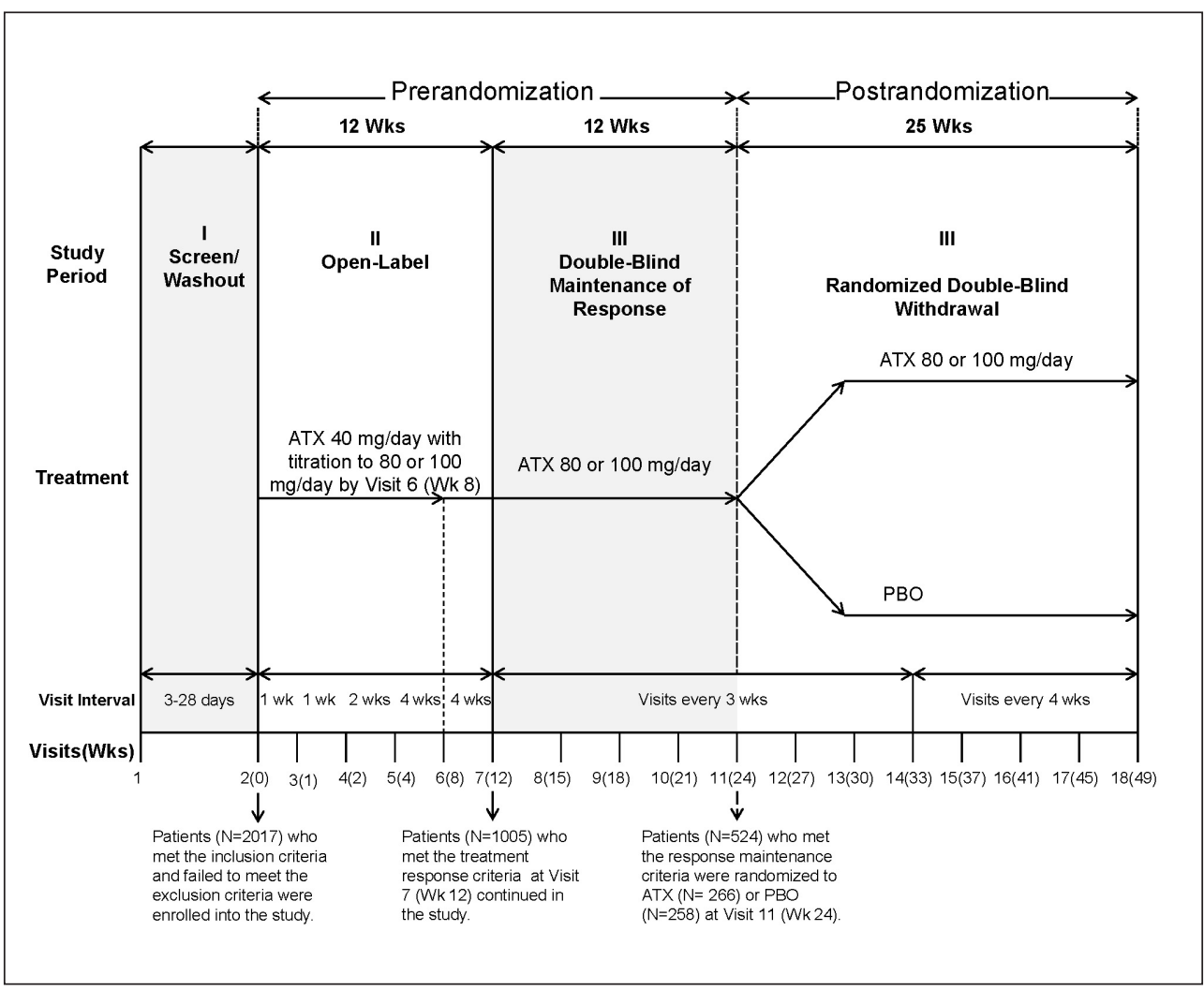

Figure 1. Illustration of study design.

Abbreviations: $\mathrm{ATX}=$ atomoxetine; $\mathrm{PBO}=$ placebo $; \mathrm{Wks}=$ weeks .

The first study period was a screening/ washout phase. The second study period was a 12-week open-label atomoxetine -treatment phase (Visits 2 to 7 [Weeks 0 to 12]). During this study period, patients received open-label atomoxetine at a starting dose of $40 \mathrm{mg} /$ day (Visit 2 [Week 0]) after completion of all assessments, with titration to a target dose of 80 or $100 \mathrm{mg} /$ day (depending on tolerability) by Visit 6 (Week 8). This dose was maintained up to Visit 7 (Week 12). At Week 12, patients were classified as responders if they had a $\geq 30 \%$ reduction from their baseline (Visit 2) CAARS-Inv:SV 18-item total ADHD symptom score, and a CGI-ADHD-S score $\leq 3$ (minimally ill). These criteria are commonly used in ADHD studies and provide a clinically meaningful level of improvement ${ }^{30,31}$.

The third study period began with a 12 week (Visits 7 to 11 [Weeks 12 to 24]) double-blind maintenance phase with atomoxetine (80 or 
$100 \mathrm{mg} /$ day). Only atomoxetine responders were allowed to enter this study period; nonresponders were discontinued from the study. During this study period, atomoxetine responders were required to continuously maintain their initial treatment responses for 3 months after assessment at Visit 7 (Week 12) and through assessment at Visit 11 (Week 24), except for 1 excursion. An "excursion" was a brief shift in the patient's symptoms or behavior that did not affect the core ADHD symptoms, but was reflected in the patient's overall CGI-ADHD-S score. An excursion was defined as a change (worsening) in the CGI-ADHD-S $\leq 1$ point from the patient's Visit 7 score, not to exceed a score of 4 (moderately ill) and a CAARS-Inv:SV total ADHD symptom score that still met the response criterion (reduction $\geq 30 \%$ in the baseline [Visit 2] score).

The last portion of the third study period was a 25-week, double-blind, randomised withdrawal phase where atomoxetine responders who were able to maintain their treatment response were randomised in a 1:1 ratio to atomoxetine ( 80 or $100 \mathrm{mg} /$ day) or placebo. During this study period, patients were required to have continuously met the response criteria for 25 weeks, after assessment at Visit 11 (Week 24) and through assessment at Visit 18 (Week 49), except for a total of 2 excursions. Patients were allowed one excursion after assessment at Week 24 and through assessment at Week 37 (13 weeks) and another excursion after assessment at Week 37 and through assessment at Week 49 (12 weeks). Patients were not permitted to have excursions at two consecutive visits. To be considered a relapser, patients must have had two consecutive visits with a CGI-ADHD-S score $\geq 4$ points and a return to $\geq 80 \%$ of the patient's baseline (Visit 2) CAARS-Inv:SV total score after Visit 11 (Week 24). If a patient showed evidence of a return of symptoms at any single visit that met the severity criteria described above and, because of worsening symptoms, was unwilling to remain in the study or did not return for a second visit, the patient was also considered to have relapsed and was discontinued from the study.

\section{Measures}

The primary efficacy measure was maintenance of response during the double-blind, randomized withdrawal phase. Time-to-relapse was a secondary efficacy measure. Other secondary efficacy measures, in order of importance, included the following: CAARS-Inv:SV; CGI-ADHD-S; Adult Attention-Deficit/Hyperactivity Disorder Quality of Life (AAQoL) ${ }^{32}$; the CAARS-O:SV; the CAARS-Self Report: Screening Version (CAARS-S:SV) ${ }^{28}$; and EuroQol-5 Dimensions Questionnaire (EQ-5D) ${ }^{33}$ with a visual analog scale (VAS). The CAARS-Inv:SV and CGIADHD-S were collected at every visit. The remaining scales were collected at baseline (Visits 1 or 2), Visit 7, Visit 15, and the final study visit. Note that the Behavior Rating Inventory of Executive Function (BRIEF)-Adult Version: Self Report ${ }^{34}$ and the BRIEF-Adult Version: Informant ${ }^{34}$ were also collected; results will be reported elsewhere. Raters were trained according to established procedures to standardise rating practices before starting the study ${ }^{9}$. These scales were administered and scored by qualified personnel at each site.

The CAARS-Inv:SV is a 30 -item scale containing three subscales (inattention, hyperactivity-impulsivity, and the ADHD index). The 18-item total ADHD symptom score (the sum of the inattention and hyperactivity/impulsivity subscales, hereafter referred to as the total score) was one of the primary efficacy measures for response, while the other subscale scores were secondary ef- 
ficacy measures. Each individual item was scored on a 4-point scale ( 0 , not at all or never; 1 , just a little or once in awhile; 2 , pretty much or often; and 3 , very much or very frequently). The ADHD index is a 12-item subscale of the CAARS-Inv:SV. All translations of CAARS-Inv:SV with adult prompts were validated.

The CGI-ADHD-S was administered by the clinician to assess the severity of ADHD in relation to the clinician's total experience with patients having ADHD. The CGIADHD-S is a single-item rating provided from a 7-point scale with 1 meaning "normal, not at all ill" and 7 meaning "among the most extremely ill".

The CAARS-O:SV and CAARS-S:SV are the same scales as the CAARS-Inv:SV, except that the CAARS-O:SV was completed by a third-party observer of the patient (typically a significant other or close friend) and the CAARS-S:SV was completed by the patient. There was no requirement that results from the observer-rated scale be obtained in person, but there was a provision that the observer's identity and relationship to the patient be documented.

The AAQoL is a patient-reported outcome measure that was used to examine the disease-specific functional impairments relevant to adults with ADHD. The domains included in the AAQoL are work functioning; family relationships; social functioning; activities of daily living; and psychological adaptation. Higher scores on the AAQoL indicate better functioning. The EQ-5D is a 5item patient-completed scale used to assess the patient's health utility (mobility, selfcare, usual activities, pain and discomfort, and depression/anxiety). Scoring is on a 3point scale (no problems; some/moderate problems; or extreme problems). A Quality of Life "thermometer" VAS rated from 0 to 100 was presented with the EQ-5D.

\section{Power}

This study was powered to address the primary objective (atomoxetine is superior to placebo in maintaining a satisfactory response in responders at the end of a double-blind, randomised withdrawal treatment period). It was determined that approximately 2000 patients were required to start open-label atomoxetine to obtain $\geq 500$ patients for randomisation into double-blind treatment (250 to atomoxetine, 250 to placebo). This assumption was based on a $40 \%$ response rate during 12 weeks of open-label atomoxetine, and a $65 \%$ response maintenance rate during 12 weeks of double-blind maintenance with atomoxetine. The assumption also takes into account patients who were discontinued due to severe protocol violations. This sample size has $95 \%$ power to detect a difference in the proportions of response, assuming response rates of 0.43 and 0.27 for atomoxetine and placebo, respectively, using Fisher's exact test at a 2-sided, 0.05 significance level.

\section{Statistical Analyses}

Data were analysed for the first two study periods combined (i.e., open-label treatment combined with double-blind maintenance [24 weeks atomoxetine]) and for the randomised withdrawal period (i.e., double-blind treatment [25 weeks atomoxetine or placebo]). For analyses of the first 24 weeks of atomoxetine treatment, baseline was considered to be the last available observation of Visits 1 and 2. For analyses of the 25-week double-blind randomised withdrawal period, baseline was considered to be the last measurement obtained on or prior to the randomisation visit.

Significance tests for categorical patient demographics and characteristics were based on Fisher's exact test and, for continuous variables, a t-test. Exposure to study drug 
was summarised overall and by visit prior to and after double-blind treatment. For all prerandomisation efficacy measures, leastsquares means (LSmean) changes from baseline to last observation carried forward (LOCF) endpoint were analysed using an analysis of covariance (ANCOVA) with terms for baseline and investigator. Wilcoxon signed-rank tests were used to assess withingroup changes from baseline.

A Fisher's exact test was used to compare proportions of responders and relapsers between treatment groups postrandomisation. The Kaplan-Meier method ${ }^{35}$ was used to estimate the survival curves for time-to-relapse and time-to-first-excursion, and comparisons between treatment groups were conducted by a 2 -sided log-rank test. A post hoc proportional hazards regression was used to calculate hazard ratios for time-to-relapse. A mixed-effects model repeated measures (MMRM) analysis, containing the terms treatment, investigator, visit, baseline, and treatment-by-visit, was used to compare the CAARS-Inv:SV and CGI-ADHD-S treatment differences. For all other measures collected during the double-blind randomized withdrawal period, treatment differences for LSmean changes from baseline to an LOCF interval endpoint were analysed using an ANCOVA. The first LOCF (LOCF-1) was an interval from 0 to 3 months postrandomisation (Visits 12 to 15 ), whereas the second LOCF (LOCF-2) was from an interval 3 to 6 months postrandomisation (Visits 16 to 18 ). For both intervals, baseline was the point of randomisation (Visit 11). Note that the last observation from LOCF-1 was not carried forward to LOCF-2. Significance tests were based on LSMean and Type III sums-ofsquares. Subgroup comparisons were made by geographic region for each measure only if the number of patients in each subgroup was $\geq 10$.
All tests were 2-sided and conducted at the 0.05 alpha level. All statistical analyses were performed using SAS, version 9.2 (SAS Institute, Inc., Cary, NC, USA). These analyses were performed on an intent-to-treat basis.

\section{Results}

\section{Disposition, Demographics, and Treatment Adherence}

Of the 2534 patients screened, 2017 were enrolled into the study and 517 failed screening (Figure 2). Of the 2017 enrolled patients, 1493 discontinued from the first two study periods combined (open-label atomoxetine plus double-blind maintenance) for reasons such as adverse event (17.3\%); response maintenance criteria not met $(16.0 \%)$; subject decision (12.1\%); protocol violation (11.4\%); and lost to follow-up (7.4\%). In total, 524 patients were randomised to atomoxetine $(\mathrm{N}=266)$ or placebo $(\mathrm{N}=258)$ for a 25 -week double-blind randomised withdrawal phase. Of these, $184(69.2 \%)$ atomoxetine-treated patients and $165(64.0 \%)$ placebo-treated patients completed the study. Reasons for discontinuation during double-blind treatment were similar between the atomoxetine- and placebo-treatment groups with the exceptions of lack of efficacy $(1.1 \%$ vs. $4.3 \%, p=$ $0.03)$ and response maintenance criteria not being met $(0.0 \%$ vs. $1.9 \%, p=0.03)$.

Patient demographics and baseline characteristics were similar between the treatment groups at the time of randomisation (Table 1). Most patients were White, male, and from Europe. The mean age was 33.2 $(\mathrm{SD}=9.1)$ years.

Overall treatment adherence, defined as taking the prescribed medication at the prescribed dose on at least $70 \%$ of the days in 


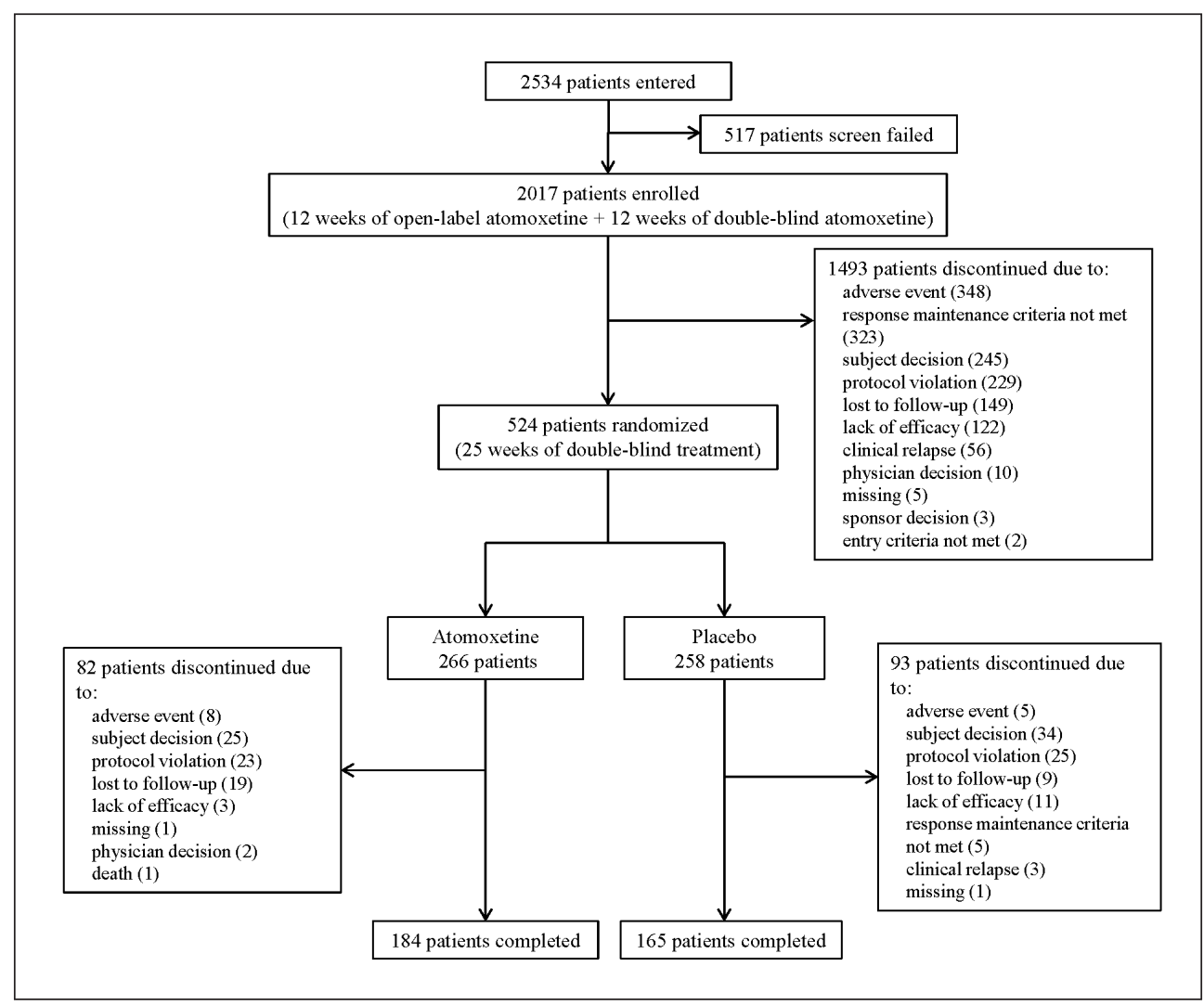

Figure 2. Subject disposition.

the visit interval, was $71.4 \%$ after 24 weeks of atomoxetine treatment and was similar between atomoxetine and placebo during the double-blind, randomized withdrawal phase (82.9\% vs. $81.0 \%$; $p=0.73$ ). Of the 2017 enrolled patients, 2011 received $\geq 1$ dose of atomoxetine. Mean exposure to atomoxetine was 105.2 days during the open-label and double-blind maintenance phases combined, and 158.9 days during the double-blind, randomised withdrawal phase.

\section{Open-Label and Double-Blind Maintenance Periods}

Core ADHD symptoms were significantly reduced after 24 weeks of atomoxetine treatment (12 weeks of open-label treatment plus 12 weeks of double-blind maintenance), as measured by the LSmean (standard error [SE]) change from baseline to LOCF in the CAARS-Inv:SV total $(-18.3$ [0.32]; $p<0.001)$, inattention $(-9.6[0.18] ; p<0.001)$, hyperactivity/impulsivity $(-8.7[0.16] ; p<0.001)$, and index $(-10.5[0.20] ; p<0.001)$ scores. These results were further supported by signifi- 


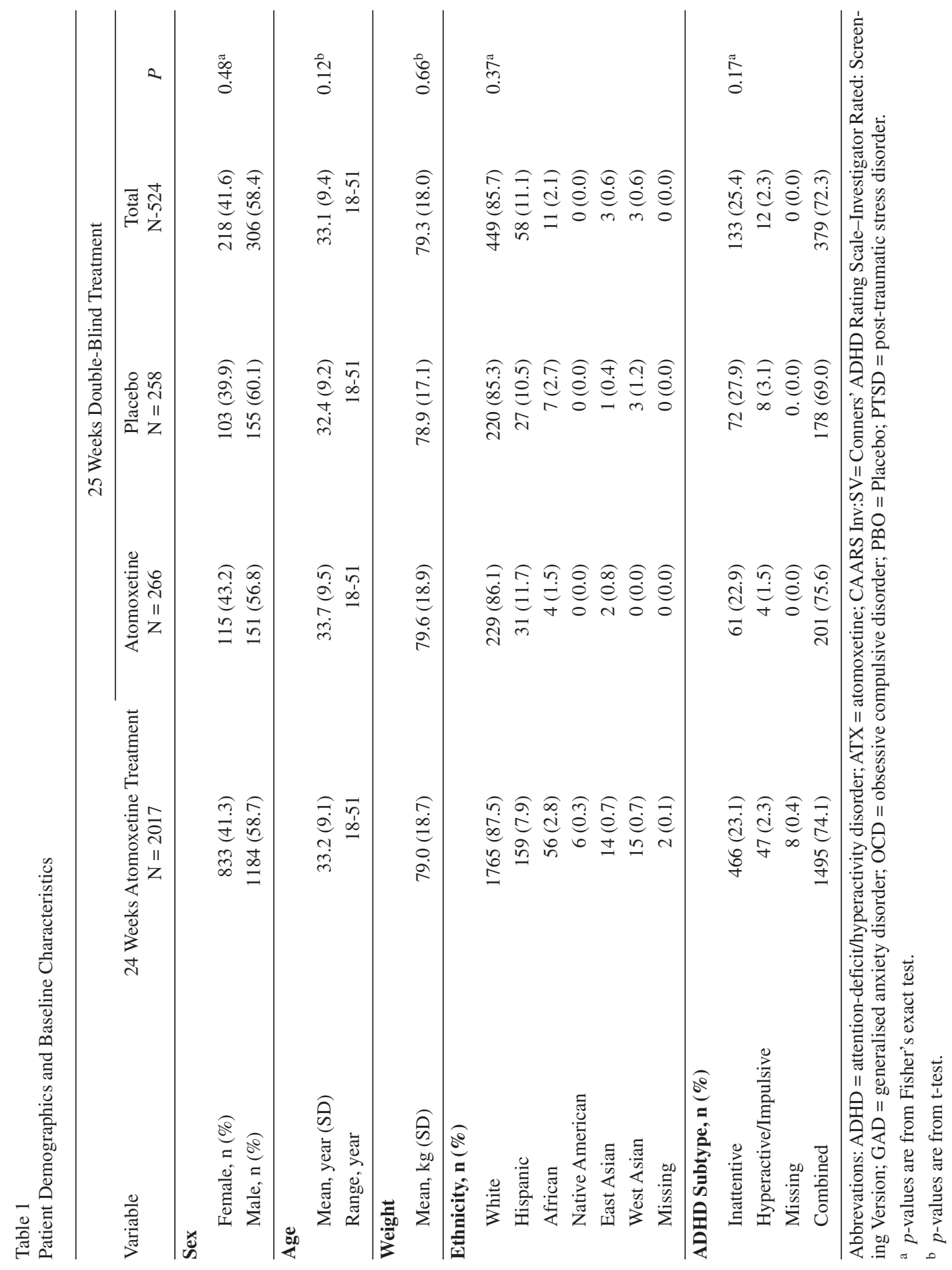




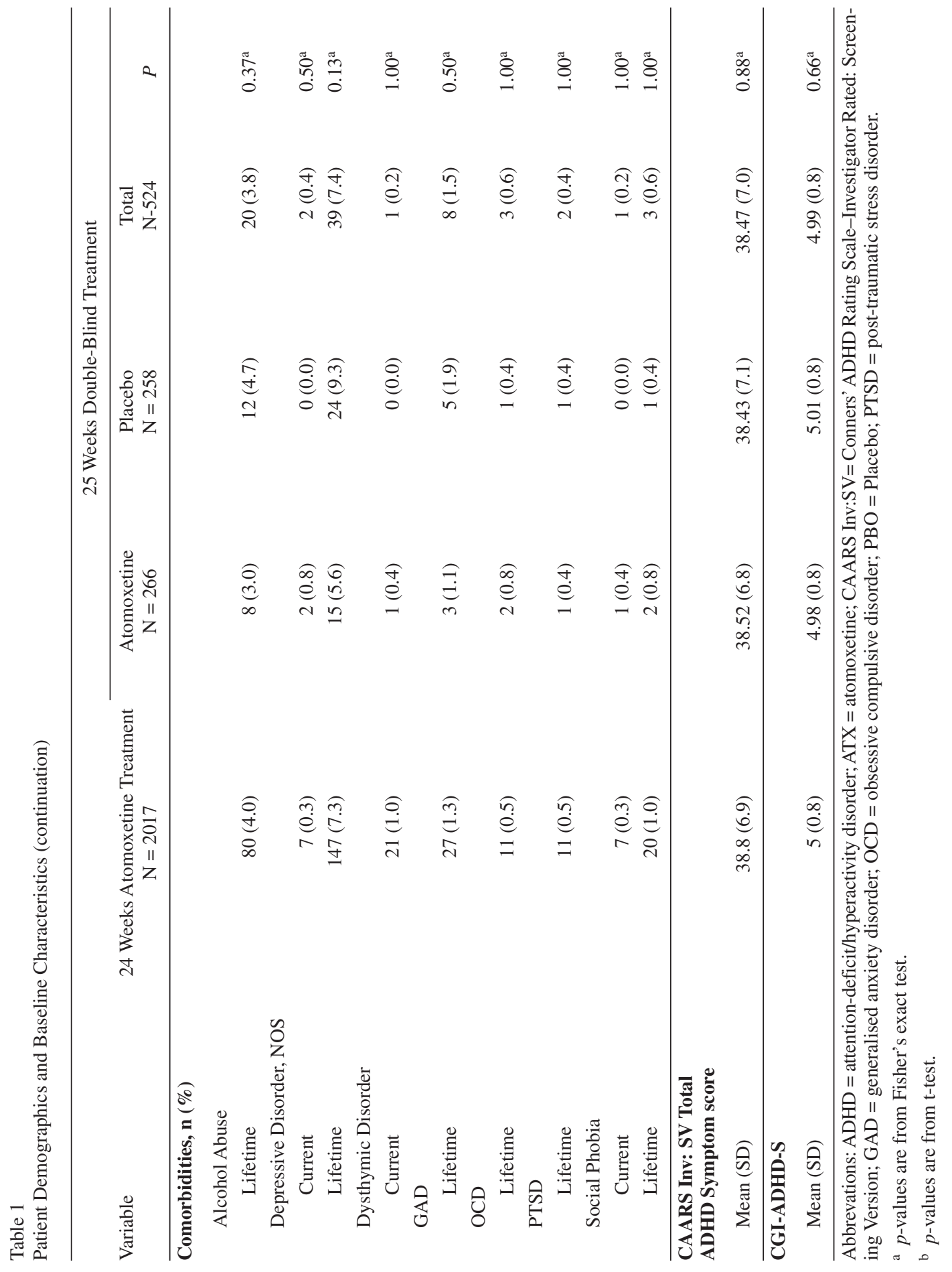


Table 2

Least-squares Mean Change from Baseline to Endpoint During 24 Weeks of Atomoxetine Treatment (12 Week Open-Label and 12 Week Double-Blind Maintenance Periods) in Secondary Efficacy Measures

Measure

Total Score

Subscale Score Baseline Endpoint Change to Endpoint

\begin{tabular}{|c|c|c|c|c|c|}
\hline & & \multirow[b]{2}{*}{$p^{b}$} \\
\hline & Mean (SD) & Mean (SD) & Mean (SD) & LSMeana (SE) & \\
\hline \multicolumn{6}{|l|}{ AAQoL } \\
\hline Total & $47.9(14.3)$ & $60.3(17.6)$ & $12.4(17.8)$ & $13.8(0.5)$ & $<0.001$ \\
\hline Outlook & $48.0(16.8)$ & $54.9(19.4)$ & $6.9(19.2)$ & $8.1(0.5)$ & $<0.001$ \\
\hline Productivity & $43.1(18.6)$ & $60.5(22.3)$ & $17.5(22.5)$ & $19.1(0.6)$ & $<0.001$ \\
\hline Psychological Health & $51.8(20.4)$ & $62.0(22.2)$ & $10.3(22.7)$ & $11.6(0.6)$ & $<0.001$ \\
\hline Life Relationships & $53.9(20.6)$ & $65.0(20.7)$ & $11.1(23.0)$ & $12.3(0.6)$ & $<0.001$ \\
\hline \multicolumn{6}{|l|}{ CAARS-O:SV } \\
\hline Total & $34.8(8.7)$ & $23.7(10.7)$ & $-11.1(11.4)$ & $-11.6(0.3)$ & $<0.001$ \\
\hline Inattention & $18.8(4.9)$ & $12.9(6.1)$ & $-5.9(6.3)$ & $-6.2(0.2)$ & $<0.001$ \\
\hline Hyperactivity/Impulsivity & $15.9(5.8)$ & $10.7(5.9)$ & $-5.2(6.3)$ & $-5.4(0.2)$ & $<0.001$ \\
\hline Index & $23.4(5.9)$ & $16.6(7.3)$ & $-6.8(7.8)$ & $-7.2(0.2)$ & $<0.001$ \\
\hline \multicolumn{6}{|l|}{ CAARS-S:SV } \\
\hline Total & $36.4(8.1)$ & $23.6(11.0)$ & $-12.8(11.5)$ & $-13.7(0.3)$ & $<0.001$ \\
\hline Inattention & $19.8(4.4)$ & $13.0(6.2)$ & $-6.8(6.4)$ & $-7.3(0.2)$ & $<0.001$ \\
\hline Hyperactivity/Impulsivity & $16.6(5.4)$ & $10.6(5.9)$ & $-6.0(6.1)$ & $-6.4(0.2)$ & $<0.001$ \\
\hline Index & $24.1(5.3)$ & $16.3(7.3)$ & $-7.8(7.6)$ & $-8.4(0.2)$ & $<0.001$ \\
\hline \multicolumn{6}{|c|}{ EQ-5D and Visual Analog Scale } \\
\hline Health State & $71.9(22.2)$ & $74.0(21.0)$ & $2.2(22.7)$ & $2.0(0.6)$ & $<0.001$ \\
\hline Mobility & $1.1(0.3)$ & $1.0(0.2)$ & $0.0(0.3)$ & $0.0(0.0)$ & 0.06 \\
\hline Self Care & $1.0(0.2)$ & $1.0(0.3)$ & $0.0(0.3)$ & $0.0(0.0)$ & 0.10 \\
\hline Usual Activities & $1.5(0.6)$ & $1.3(0.5)$ & $-0.1(0.7)$ & $-0.2(0.0)$ & $<0.001$ \\
\hline Pain/Discomfort & $1.3(0.6)$ & $1.3(0.6)$ & $0.0(0.7)$ & $0.0(0.0)$ & 0.21 \\
\hline Anxiety/Depression & $1.5(0.7)$ & $1.4(0.6)$ & $-0.1(0.8)$ & $-0.1(0.0)$ & $<0.001$ \\
\hline UK Index & $0.8(0.2)$ & $0.8(0.2)$ & $0.0(0.2)$ & $0.0(0.0)$ & $<0.001$ \\
\hline US Index & $0.8(0.1)$ & $0.9(0.1)$ & $0.0(0.2)$ & $0.0(0.0)$ & $<0.001$ \\
\hline
\end{tabular}

Abbreviations: AAQoL = Adult Attention-Deficit/Hyperactivity Disorder Quality of Life; ANCOVA = analysis of covariance; EQ-5D = EuroQol-5 Dimensions Questionnaire; CAARS-O:SV = Conners' ADHD Rating Scale-Observer Rated: Screening Version; CAARS-S:SV = Conners'ADHD Rating Scale-Self Report: Screening Version; $\mathrm{LOCF}=$ last observation carried forward; LSMean = least-squares means; $\mathrm{N}=$ number of patients with a nonmissing baseline and at least one postbaseline result within each group; $\mathrm{SD}=$ standard deviations; $\mathrm{SE}=$ standard error.

${ }^{a}$ Least-squares means (LSmean) change from baseline to last observation carried forward (LOCF) endpoint were analysed using an analysis of covariance (ANCOVA) with terms for baseline and investigator.

b $p$-values are from Wilcoxon's signed-rank test. 
cantly reduced LSmean changes from baseline to LOCF in the CAARS-O:SV and CAARS-S:SV total and subscale scores after 24 weeks of atomoxetine treatment (Table $2 ; p<0.001$ for all). Atomoxetine decreased ADHD severity after 24 weeks of treatment as measured by the LSmean (SE) change from baseline to LOCF in the CGI-ADHD-S ratings $(-1.7[0.04] ; p<0.001)$.

Quality of life was significantly improved after 24 weeks of atomoxetine treatment as shown by the LSmean (SE) change from baseline to LOCF endpoint in the AAQoL total and subscale scores (Table 2; $p<0.001$ for all). For the EQ-5D health state and subscale scores, atomoxetine showed improvements in the health state $(p<0.001)$, usual activities $(p$ $<0.001)$, and anxiety/depression $(p<0.001)$ scores after 24 weeks of atomoxetine treatment.

\section{Double-Blind Randomised Withdrawal Period}

Significantly more atomoxetine- than placebo-treated patients maintained a satisfactory response overall (64.3\% vs. 50.0\%; $p$ $<0.001)$ and by visit ( $p<.05$; data not shown) 25 weeks after randomisation. A sensitivity analysis on a subset of patients who did not have prespecified protocol violations yielded similar results overall (66.0\% vs. $50.6 \%, p$ $<0.001$ ) and by visit (data not shown). Fewer atomoxetine- than placebo-treated patients relapsed $(2.3 \%$ vs. $7.4 \%, p=0.007$; hazard ratio $=0.355, p=0.03$ ), and time-to-relapse and time-to-first excursion were significantly longer for atomoxetine- than placebo-treated patients, as determined by Kaplan-Meier survival curves ( $p=0.004$ and $p<0.001$, respectively; see Figure 3 for time-to-relapse during

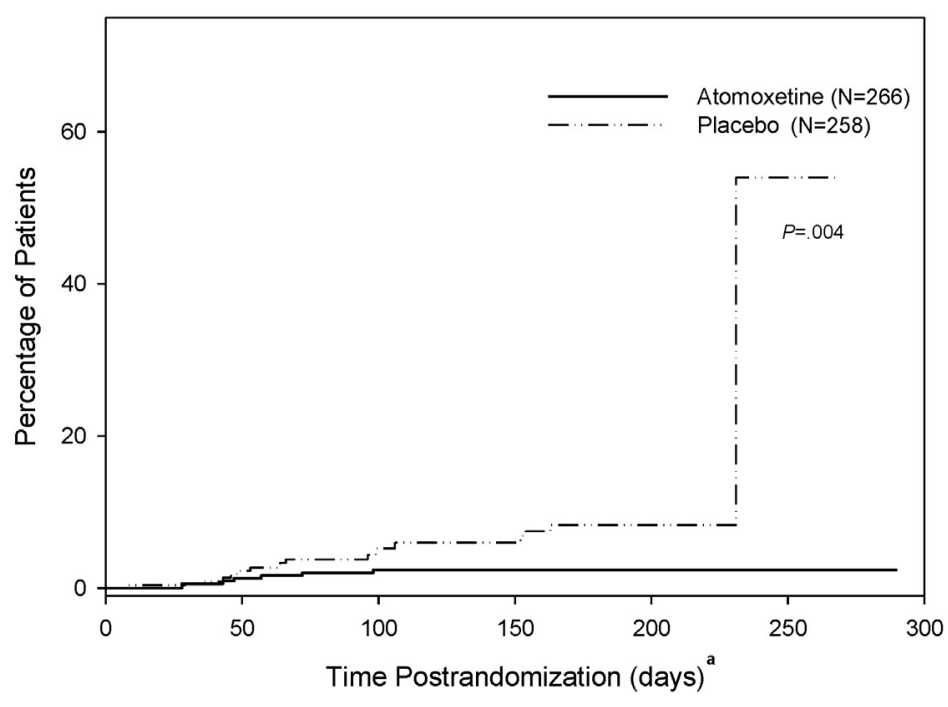

Figure 3. Kaplan-Meier plot with the percentage of patients on the vertical axis, and the time-to-relapse variable along the horizontal axis for patients with ADHD randomly assigned to atomoxetine $(\mathrm{N}=266)$ or placebo $(\mathrm{N}=258)$ for 25 weeks of double-blind treatment.

a The duration of the double-blind randomized withdrawal phase was 25 weeks (approximately 180 days). Few patients stayed longer than 25 weeks during this phase; therefore at Day 230, there appears to be a dramatic increase in relapse due to 1 out of 2 remaining patients relapsing. 
the 25 week, double-blind, randomised withdrawal phase).

Significantly lower LSMean worsening (lower symptom severity) in the CAARSInv:SV total score and subscale scores were observed with atomoxetine than with placebo at Visit 18 (Week 49 [0.9 vs. 4.8; $p<0.001]$ ), and every other visit during the 25 -week, double-blind, randomised withdrawal period as shown by MMRM analyses in Figure 4 (visit-wise $p<0.001$ ).

These results were further supported by significantly lower LSMean worsening from baseline to LOCF-2 in the CAARS-O:SV and CAARS-S:SV total and subscale scores with atomoxetine than with placebo (Table 3; $p<0.01$ for all). Analyses of the LSMean changes from baseline to LOCF-1 in the CAARS-O:SV and CAARS-S:SV total and subscale scores (data not shown) yielded similar results to analyses of LOCF-2 shown in Table 3. Significantly lower (decrease in overall ADHD severity) LSMean worsening from baseline in the CGI-ADHD-S was observed with atomoxetine than with placebo at Visit 18 (Week 49 [0.0 vs. $0.5 ; p<0.001]$ ) and at every other visit postrandomisation as shown by the MMRM analysis in Figure 5 (visit-wise $p<0.001$ ). There were no significant differences between geographic regions for any of these measures.

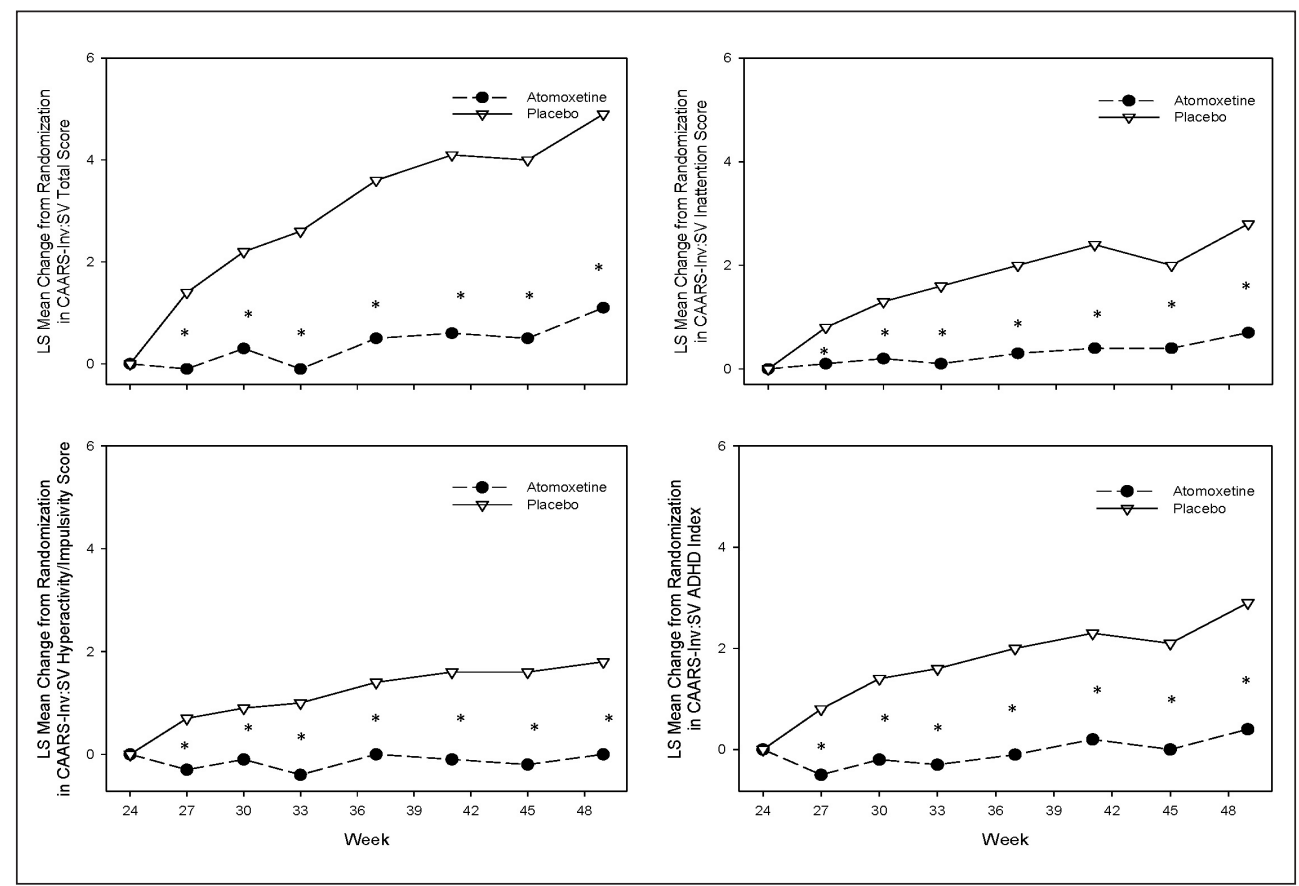

Figure 4. Least-squares mean change of the Conners' Adult Attention-Deficit/Hyperactivity Disorder Rating Scale-Investigator Screening Version total, hyperactivity/impulsivity, inattention, and index scores from mixed-effects model repeated measures among patients with ADHD randomly assigned to atomoxetine $(\mathrm{N}=266)$ or placebo $(\mathrm{N}=258)$ for 25 weeks of double-blind treatment.

Abbreviations: ADHD = attention-deficit/hyperactivity disorder; CAARS-Inv:SV = Conners' Adult Attention-Deficit/Hyperactivity Disorder Rating Scale-Investigator Screening Version; CAARS-Inv:SV Total Score = CAARS-Inv:SV 18-item ADHD Total Symptom Score.

$* p<0.001$. 


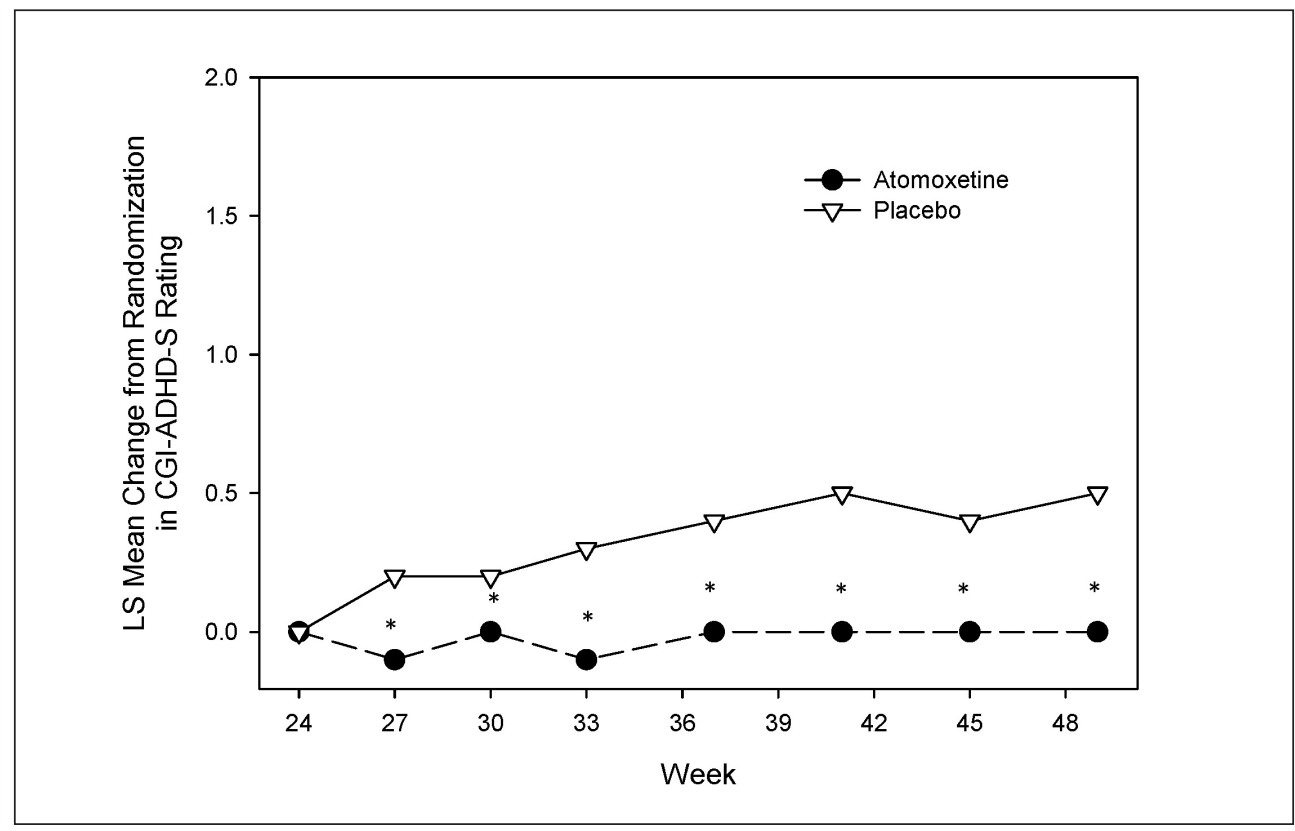

Figure 5. Least-squares mean change of the CGI-ADHD-Severity ratings from mixed-effects model repeated measures among patients with ADHD randomly assigned to atomoxetine $(\mathrm{N}=266)$ or placebo $(\mathrm{N}=258)$ for 25 weeks of double-blind treatment.

Abbreviations: $\mathrm{ADHD}=$ attention-deficit/ hyperactivity disorder; CGI-ADHD-S = Clinical Global Impression ADHD Severity. $* p<0.001$.

Improvements in quality of life measured during the first 24 weeks of atomoxetine treatment were better maintained with atomoxetine than with placebo during the 25week, double-blind, randomized withdrawal period as shown by significant LSmean changes from baseline to LOCF-2 endpoint in the AAQoL total (Table 3; $p=0.002$ ), productivity $(p<0.001)$ and psychological health $(p=0.003)$ scores. Although atomoxetine showed improvements in the EQ-5D health state score and other scores during the first 24 weeks of atomoxetine treatment, there were no significant differences between atomoxetine and placebo 25 weeks postrandomisation. Analyses of the LSMean changes from baseline to LOCF-1 in the AAQoL and the EQ-5D (data not shown) yielded similar re- sults to analyses of LOCF-2 shown in Table 3. Of the subgroup analyses by geographic region, the EQ-5D, but not the AAQoL, had statistically significant treatment-by-subgroup interactions for country on the mobility $(p=0.02)$ and self-care scores $(p=0.02)$ at LOCF-2, both of which were not clinically meaningful because there were very few observations for multiple countries.

\section{Discussion}

To our knowledge, the data presented here are from the largest clinical trial of an ADHD medication (pediatric or adult) performed to date and demonstrate that atomoxetine, at 


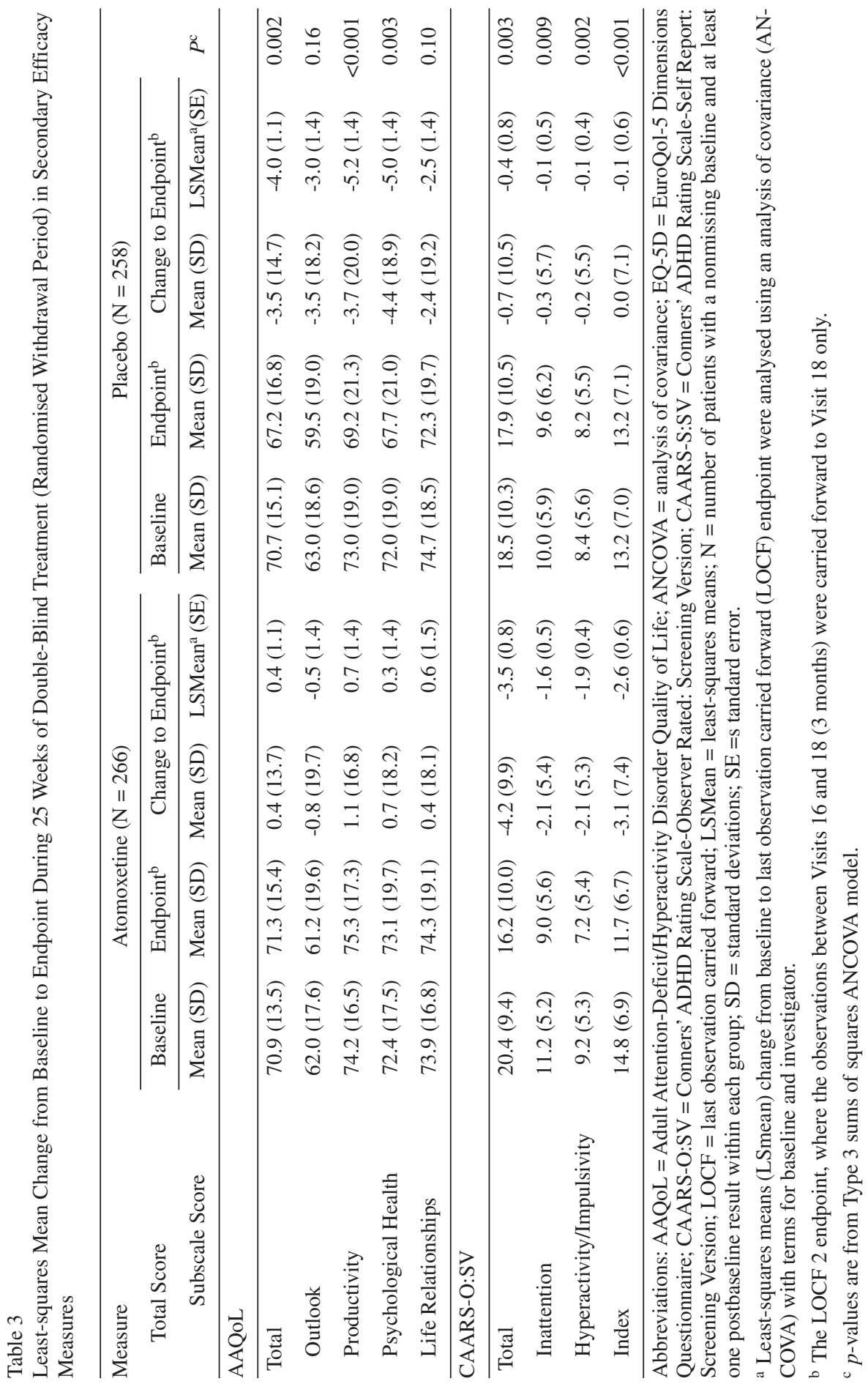




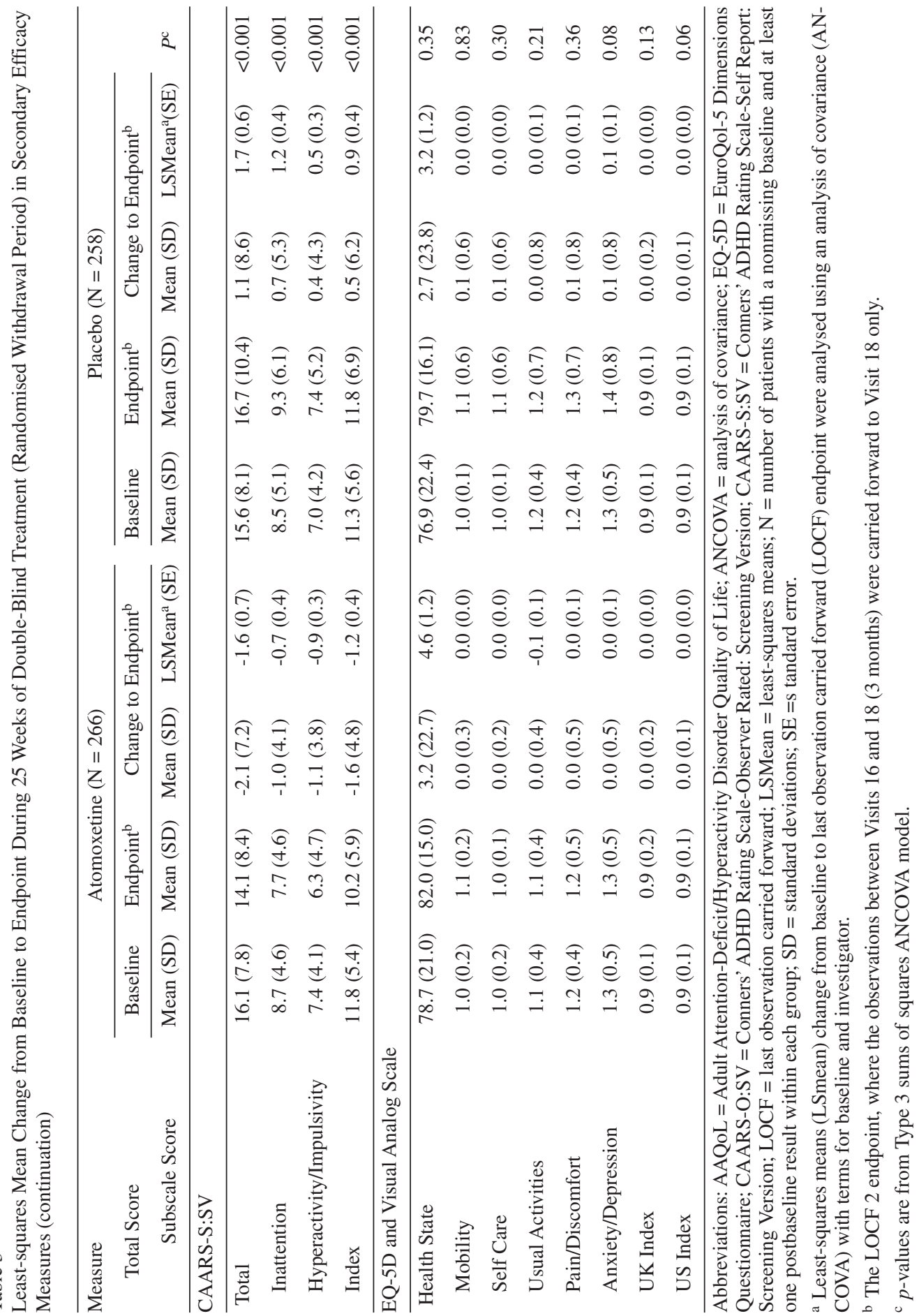


doses of 80 or $100 \mathrm{mg} /$ day, maintains superior treatment responses for 25 weeks in adult patients with ADHD who initially responded to 24 weeks of atomoxetine treatment during a 12-week open-label treatment period and a 12-week double-blind maintenance period. Furthermore, this study extends the findings of previous studies of atomoxetine in adults ${ }^{7,9,10}$ by providing evidence of the efficacy of atomoxetine in a large international, multicenter sample. The findings are also consistent with relapse prevention and maintenance studies in children and adolescents treated with atomoxetine ${ }^{23-25}$. In the current study, atomoxetine showed significant amelioration of ADHD symptoms (47\% [18.3 point] improvement) as measured by the CAARS-Inv:SV and CGI-ADHD-S after 24 weeks of initial treatment, which were maintained during an additional 25 weeks of double-blind treatment. These results were further supported by self- or observer-rated measures (CAARS-O:SV and CAARS-S:SV) and were consistent when evaluating total scores, which included the mixed subtype, or when separating out results by the hyperactivity/impulsivity or inattentive ADHD subtypes.

Effective control of ADHD symptoms for the long term may provide ADHD patients with the opportunity to develop better adaptive skills that could continue to offer benefits even in the absence of active treatment. In the current study, ADHD symptoms were effectively controlled by atomoxetine as witnessed by a significantly greater proportion of atomoxetine- than placebo-treated patients maintaining a satisfactory response $(\geq 30 \%$ continuous reduction from baseline in CAARS-Inv:SV total score and a rating $\leq 3$ on the CGI-ADHD-S). Although significantly more placebo- than atomoxetinetreated patients relapsed, the proportion of placebo-relapsers was relatively small, suggesting that atomoxetine treatment for 24 weeks prerandomisation may have continued to offer benefits after the cessation of treatment. For example, there may have been neurobehavioral and neurobiological adaptations and environmental changes with improved ADHD symptoms over a period of time such that the placebo-treated patients may not relapse immediately upon discontinuation of atomoxetine. This is supported by evidence that normalization of brain structures may occur with improved ADHD symptoms (as found in study periods averaging 2 years $)^{36,37}$. In addition, there could be positive changes to the patient's environment, such as how others view and interact with the patient. Positive changes in the patient's environment may, in turn, decrease the chance of relapse: the patient feels better about his abilities to overcome symptoms and, because of earlier success, feels more confident to and can work through the return of some symptoms rather than relapse. It is possible that the long-term (6 month) successful treatment with atomoxetine before being randomized to placebo led to such behavioral and environmental changes that accentuated the response to placebo during the maintenance of response phase.

The lower relapse rate after long term treatment with atomoxetine seen in the current study is consistent with results from relapse prevention studies in children and adolescents aged 6 to 15 years, where relapse criteria was defined as a symptom return to $90 \%$ or greater of the baseline ADHD-Rating Scale-IV total score and an increase in the CGI-ADHD-S score of at least 2 points, during the relapse prevention phase ${ }^{24,25}$. Relapse rates for pediatric patients after one year of successful treatment with atomoxetine were lower $(12.2 \%)^{25}$ than after 10 weeks of successful treatment with atomoxetine $(37.9 \%)^{24}$, suggesting that longer periods of treatment with atomoxetine that achieve a satisfactory response may lead 
to lower relapse rates perhaps due to neurobehavioral adaptation.

The low relapse rate for placebo-treated patients, however, could also be due in part to the stringent criteria used for relapse in this study: Patients were required to have had two consecutive visits with a CGI-ADHD-S score $\geq 4$ points and a return to $\geq 80 \%$ of the patient's baseline (Visit 2) CAARSnv:SV total score after Visit 11 (Week 24). In contrast to the $80 \%$ return to baseline symptom criterion used for relapse in this study, a range of 50 to $80 \%$ return to baseline symptom has been used as a criterion for relapse in the literature $^{8,38,39}$. The low relapse rates in the current study could also be caused by other factors such as nonspecific benefits related to continued participation and ongoing medical contact. Regardless of the cause, the placebo results in this study collectively suggest that, after drug discontinuation, patients may experience return of some symptoms, and that continued treatment with atomoxetine has a significant protective effect against this.

The current study provides evidence that improvements in core ADHD symptoms are associated with sustained improvements in quality of life. Atomoxetine significantly improved quality of life after 24 weeks of treatment as measured by the AAQoL; these improvements were maintained throughout the study. Atomoxetine also significantly improved health utility as measured by the EQ5D; however, this improvement was not maintained during double-blind treatment. This may be caused, in part, by the fact that 4 of 5 scales on the EQ-5D measure physical disability, not psychological impairment. Hence, the EQ-5D may not be the most appropriate measure for ADHD. The AAQoL results in the current study are consistent with other short- and long-term studies of atomoxetine in adults ${ }^{7,40}$ and suggest that atomoxetine can improve the impairments and consequences of ADHD that negatively impact quality of life. It remains to be determined, however, whether long-term treatment of adult ADHD with atomoxetine will improve lives in terms of education, employment, family relationships, and the occurrence of traffic accidents ${ }^{2-5}$.

Although this study demonstrated atomoxetine's superior maintenance of response, several limitations should be considered when evaluating these data. Substantial sample attrition took place during the 12-week open-label and 12-week double-blind maintenance periods primarily due to reasons other than nonresponse to atomoxetine, although the completion rate was consistent with or better than long-term studies of atomoxetine ${ }^{7}$ and $\mathrm{MPH}^{8,35}$. The limitation lies in the fact that the double-blind randomised withdrawal data only describe a subpopulation that responded to atomoxetine and were compliant with the previous 24 weeks of treatment and the lengthy study protocol schedule. Those patients evolving poorly and with less behavioral adaptations before randomisation might have been more prone to relapse when stopping atomoxetine treatment, but these patients were likely to be eliminated by the interim criteria for response before randomisation. This is demonstrated by the number of discontinuations due to interim response criteria not met $(\mathrm{N}=323)$, relapse $(\mathrm{N}=56)$, and lack of efficacy $(\mathrm{N}=122)$ in the initial 6 months, or about $25 \%$ of the enrolled patients. Furthermore, because the first phase of the study was open-label, a number of patients who would have done well even without active drug treatment, such as placebo responders reacting well to educational influences, may have been selected as responders and would be less likely to relapse when active treatment was stopped. This limits generalisation of these findings to typical outpatients as does the fact that pa- 
tients had relatively fewer current comorbid medical conditions and concomitant medications compared with their general outpatient counterparts. Another limitation was that the response criteria were stricter than most, with patients who showed a 2-point improvement on CGI-ADHD-S being discontinued because they did not meet the protocol-defined response criteria prior to randomisation.

In summary, this study demonstrated that atomoxetine reduced ADHD symptoms and improved ADHD quality of life in adult outpatients after 24 weeks of treatment and that atomoxetine was superior to placebo in maintaining a satisfactory response after another 25 weeks of treatment. The findings support the usual clinical practice of maintaining treatment for extended periods in patients whose symptoms respond to an initial treatment period.

\section{Acknowledgements}

The trial was funded and sponsored by Eli Lilly and Company and/or any of its subsidiaries, Indianapolis, IN, USA. We would like to thank PharmaNet/i3 for their help with editing and formatting.

\section{References}

1. Fayyad J, De Graaf R, Kessler R, Alonso J, Angermeyer M, Demyttenaere K, et al. Cross-national prevalence and correlates of adult attention-deficit hyperactivity disorder. Br J Psychiatry 2007; 190: 402-409.

2. Adler L, Cohen J. Diagnosis and evaluation of adults with attention-deficit/hyperactivity disorder. Psychiatr Clin North Am 2004; 27(2): 187-201.

3. Barkley RA. Major life activity and health outcomes associated with attention-deficit/hyperactivity disorder. J Clin Psychiatry 2002; 63(12): 10-15.
4. Faraone SV, Biederman J, Spencer T, Wilens T, Seidman LJ, Mick E, et al. Attention-deficit/hyperactivity disorder in adults: an overview. Biol Psychiatr. 2000; 48(1): 9-20.

5. Fischer M, Barkley RA, Smallish L, Fletcher K. Hyperactive children as young adults: driving abilities, safe driving behavior, and adverse driving outcomes. Accid Anal Prev 2007; 39(1): 94-105.

6. McGough JJ, Smalley SL, McCracken JT, Yang M, Del'Homme M, Lynn DE, et al. Psychiatric comorbidity in adult attention deficit hyperactivity disorder: findings from multiplex families. Am J Psychiatry 2005; 162(9): 1621-1627.

7. Adler LA, Spencer T, Brown TE, Holdnack J, Saylor $\mathrm{K}$, Schuh K, et al. Once-daily atomoxetine for adult attention-deficit/hyperactivity disorder: a 6-month, double-blind trial. J Clin Psychopharmacol 2009; 29(1): 44-50.

8. Biederman J, Mick E, Surman C, Doyle R, Hammerness P, Kotarski M, et al. A randomized, 3-phase, 34-week, double-blind, long-term efficacy study of osmotic-release oral system-methylphenidate in adults with attentiondeficit/hyperactivity disorder. J Clin Psychopharmacol 2010; 30(5): 549-553.

9. Michelson D, Adler L, Spencer T, Reimherr FW, West $\mathrm{SA}$, Allen AJ, et al. Atomoxetine in adults with ADHD: two randomized, placebo-controlled studies. Biol Psychiatry 2003; 53(2): 112-120.

10. Spencer T, Biederman J, Wilens T, Prince J, Hatch M, Jones $\mathrm{J}$, et al. Effectiveness and tolerability of tomoxetine in adults with attention deficit hyperactivity disorder. Am J Psychiatry 1998; 155(5): 693-695.

11. Spencer T, Biederman J, Wilens T, Doyle R, Surman C, Prince J, et al. A large, double-blind, randomized clinical trial of methylphenidate in the treatment of adults with attention deficit/hyperactivity disorder. Biol Psychiatry. 2005; 57(5): 456-463.

12. Bouffard R, Hechtman L, Minde K, Iaboni-Kassab F. The efficacy of 2 different dosages of methylphenidate in treating adults with attention-deficit hyperactivity disorder. Can J Psychiatry 2003,48(8): 546-554.

13. Kooij JJ, Burger H, Boonstra AM, Van der Linden PD, Kalma LE, Buitelaar JK. Efficacy and safety of methylphenidate in 45 adults with attention-deficit/hyperactivity disorder. A randomized placebo-controlled double-blind crossover trial. Psychol Med 2004; 34(6): 973-982.

14. Medori R, Ramos-Quiroga JA, Casas M, Kooij JJ, Niemelä A, Trott GE, et al. A randomized, placebo-controlled trial of three fixed dosages of prolonged-release OROS methylphenidate in adults with attention-deficit/hyperactivity disorder. Biol Psychiatry 2008; 63(10): 981-989. 
15. Kooij SJ, Bejerot S, Blackwell A, Caci H, CasasBrugué M, Carpentier PJ, et al. European consensus statement on diagnosis and treatment of adult ADHD: the European network adult ADHD. BMC Psychiatry 2010; 10: 67.

16. Spencer T, Biederman J, Wilens T, Harding M, O'Donnell D, Griffin S. Pharmacotherapy of attentiondeficit hyperactivity disorder across the life cycle. J Am Acad Child Adolesc Psychiatry 1996; 35(4): 409-432.

17. Wilens TE, Biederman J. The stimulants. Psychiatr Clin North Am 1992; 15(1): 191-222.

18. Vaughan B, Fegert J, Kratochvil CJ. Update on atomoxetine in the treatment of attention-deficit/hyperactivity disorder. Expert Opin Pharmacother 2009; 10(4): 669-676.

19. Kelsey DK, Sumner CR, Casat CD, Coury DL, Quintana H, Saylor KE, et al. Once-daily atomoxetine treatment for children with attention-deficit/hyperactivity disorder, including an assessment of evening and morning behavior: a double-blind, placebo-controlled trial. Pediatrics 2004; 114(1): e1-e8.

20. Michelson D, Faries D, Wernicke J, Kelsey D, Kendrick K, Sallee FR, et al. Atomoxetine in the treatment of children and adolescents with attention-deficit/hyperactivity disorder: a randomized, placebo-controlled, dose-response study. Pediatrics 2001; 108(5): E83.

21. Michelson D, Allen AJ, Busner J, Casat C, Dunn D, Kratochvil C, et al. Once-daily atomoxetine treatment for children and adolescents with attention deficit hyperactivity disorder: a randomized, placebo-controlled study. Am J Psychiatry 2002; 159(11): 1896-1901.

22. Spencer T, Heiligenstein JH, Biederman J, Faries DE, Kratochvil CJ, Conners CK, et al. Results from 2 proofof-concept, placebo-controlled studies of atomoxetine in children with attention-deficit/hyperactivity disorder. J Clin Psychiatry 2002; 63(12): 1140-1147.

23. Newcorn JH, Michelson D, Kratochvil CJ, Allen AJ, Ruff DD, Moore RJ; Atomoxetine Low-Dose Study Group. Low-dose atomoxetine for maintenance treatment of attention-deficit/hyperactivity disorder. Pediatrics 2006; 118(6): e1701-e1706

24. Michelson D, Buitelaar JK, Danckaerts M, Gillberg C, Spencer TJ, Zuddas A, et al. Relapse prevention in pediatric patients with ADHD treated with atomoxetine: a randomized, double-blind, placebo-controlled study. J Am Acad Child Adolesc Psychiatry 2004; 43(7): 896-904.

25. Buitelaar JK, Michelson D, Danckaerts M, Gillberg C, Spencer TJ, Zuddas A, et al. A randomized, doubleblind study of continuation treatment for attention-deficit/hyperactivity disorder after 1 year. Biol Psychiatry 2007; 61(5): 694-699.
26. Wilens TE, Adler LA, Weiss MD, Michelson D, Ramsey JL, Moore RJ, et al. Atomoxetine treatment of adults with ADHD and comorbid alcohol use disorders. Drug Alcohol Depend 2008; 96(1-2): 145-154.

27. Adler LA, Liebowitz M, Kronenberger W, Qiao M, Rubin R, Hollandbeck M, et al. Atomoxetine treatment in adults with attention-deficit/hyperactivity disorder and comorbid social anxiety disorder. Depress Anxiety 2009; 26(3): 212-221.

28. Conners CK, Erhardt D, Sparrow E. Conners' Adult ADHD Rating Scales (CAARS). North Tonawanda, NY: Multi-Health Systems, Inc.; 1999.

29. Guy W. ECDEU Assessment Manual for Psychopharmacology, Revised. Bethesda, MD: US Department of Health, Education, and Welfare; 1976.

30. Adler LA, Wilens T, Zhang S, Dittmann RW, D'Souza $\mathrm{DN}$, Schuh L, et al. Atomoxetine treatment outcomes in adolescents and young adults with attention-deficit/hyperactivity disorder: results from a post hoc, pooled analysis. Clin Ther 2012; 34(2): 363-373.

31. Wilens TE, Spencer TJ, Biederman J, Girard K, Doyle $\mathrm{R}$, Prince $\mathrm{J}$, et al. A controlled clinical trial of bupropion for attention deficit hyperactivity disorder in adults. Am J Psychiatry $2001 ; 158(2)$ : 282-288.

32. Brod M, Johnston J, Able S, Swindle R. Validation of the adult attention-deficit/hyperactivity disorder quality-oflife Scale (AAQoL): a disease-specific quality-of-life measure. Qual Life Res 2006; 15(1): 117-129.

33. The EuroQol Group. EuroQol-a new facility for the measurement of health-related quality of life. Health Policy 1990; 16(3): 199-208.

34. Roth RM, Isquith PK, Gioia GA. Behavior Rating Inventory of Executive Function-Adult Version. Lutz, FL: Psychological Assessment Resources, Inc.; 2005.

35. Kaplan EL, Meier P. Nonparametric estimation from incomplete observations. J Am Stat Assoc 1958; 53: 457-481.

36. Shaw P, Gogtay N, Rapoport J. Childhood psychiatric disorders as anomalies in neurodevelopmental trajectories. Hum Brain Mapp 2010; 31(6): 917-925.

37. Nakao T, Radua J, Rubia K, Mataix-Cols D. Grey matter volume abnormalities in ADHD: voxel-based metaanalysis exploring the effects of age and stimulant medication. Am J Psychiatry 2011; 168(11): 1154-1163.

38. Buitelaar JK, Trott G-E, Hofecker M, Waechter S, Berwaerts J, Dejonkheere J, . Long-term efficacy and safety outcomes with OROS-MPH in adults with ADHD. Int $\mathrm{J}$ Neuropsychopharmacol 2012; 15(1): 1-13. 
39. Brams M, Weisler R, Findling RL, Gasior M, Hamdani M, Ferreira-Cornwell C, et al. Maintenance of efficacy of lisdexamfetamine dimesylate in adults with attentiondeficit/ hyperactivity disorder: randomized withdrawal design. J Clin Psychiatry 2012; 73(7): 977-983.

40. Adler LA, Sutton VK, Moore RJ, Dietrich AP, Reimherr FW, Sangal RB, et al. Quality of life assessment in adult patients with attention-deficit/hyperactivity disorder treated with atomoxetine. J Clin Psychopharmacol 2006; 26(6): 648-652.
Corresponding author:

Himanshu Upadhyaya, MBBS, MS

Lilly Corporate Center

Indianapolis, IN

Telephone: 1-713-592-0912

Fax: 1-713-592-0916

E-mail: upadhyayahp@1illy.com 